Supporting Information

\title{
Stretchable Carbon Nanotube Dilatometer for In-situ Swelling Detection of
} Lithium-ion Battery

Leilei Wang†, Woohyuk Choi†, Kisoo Yoo, Kanghyun Nam, Tae Jo Ko*, and Jungwook Choi*

School of Mechanical Engineering, Yeungnam University

280 Daehak-ro, Gyeongsan, Gyeongbuk 38541, Republic of Korea

$\dagger$ These authors are contributed equally to this work.

*E-mail: T. J. Ko: tjko@yu.ac.kr, J. Choi: jwc@yu.ac.kr

\section{Contents:}

1. Mechanical property of PDMS

2. Characterization of LIB used for swelling measurements

3. Characterization of CNT-sensor according to the CNT thickness

4. Mechanical and thermal reliability of CNT-sensor

5. Dependence of the coefficient of thermal expansion of PDMS on temperature

6. Noise processing using RLS algorithm

7. LIB swelling during regular charge and discharge cycles

8. Irregular, irreversible, thermomechanical swelling of LIB

9. References 


\section{Mechanical property of PDMS}

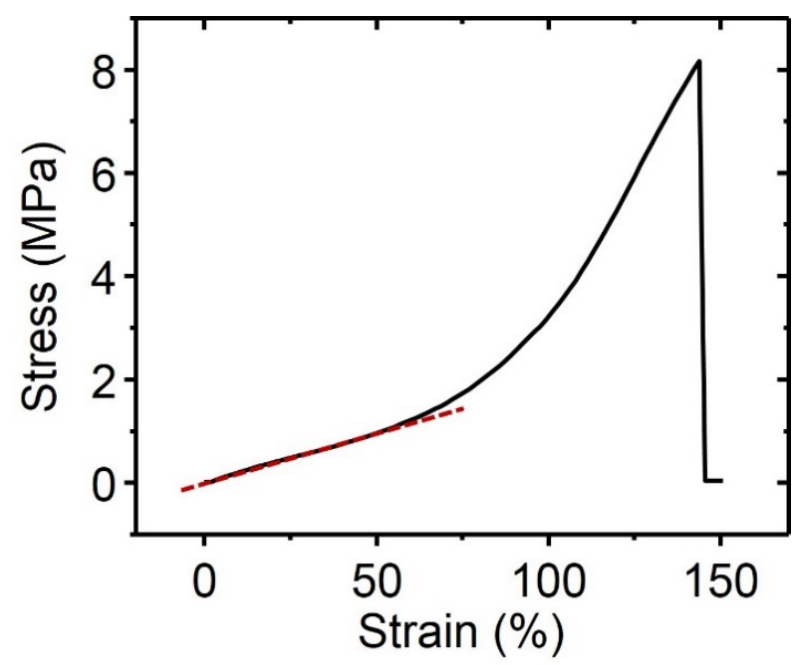

Figure S1. Tensile mechanical property of PDMS measured using a universal testing machine (LRX Plus, LLOYD) with a load rate of $5 \mathrm{~mm} / \mathrm{min}$. Initial linear slope represents the elastic modulus of PDMS (1.92 MPa). The measured elastic modulus of PDMS corresponds well with the previously reported value. ${ }^{1}$ This low elastic modulus imparts stretchability to the CNT-sensor, which is useful for detecting the swelling of the LIB while minimizing interference. 
2. Characterization of LIB used for swelling measurements
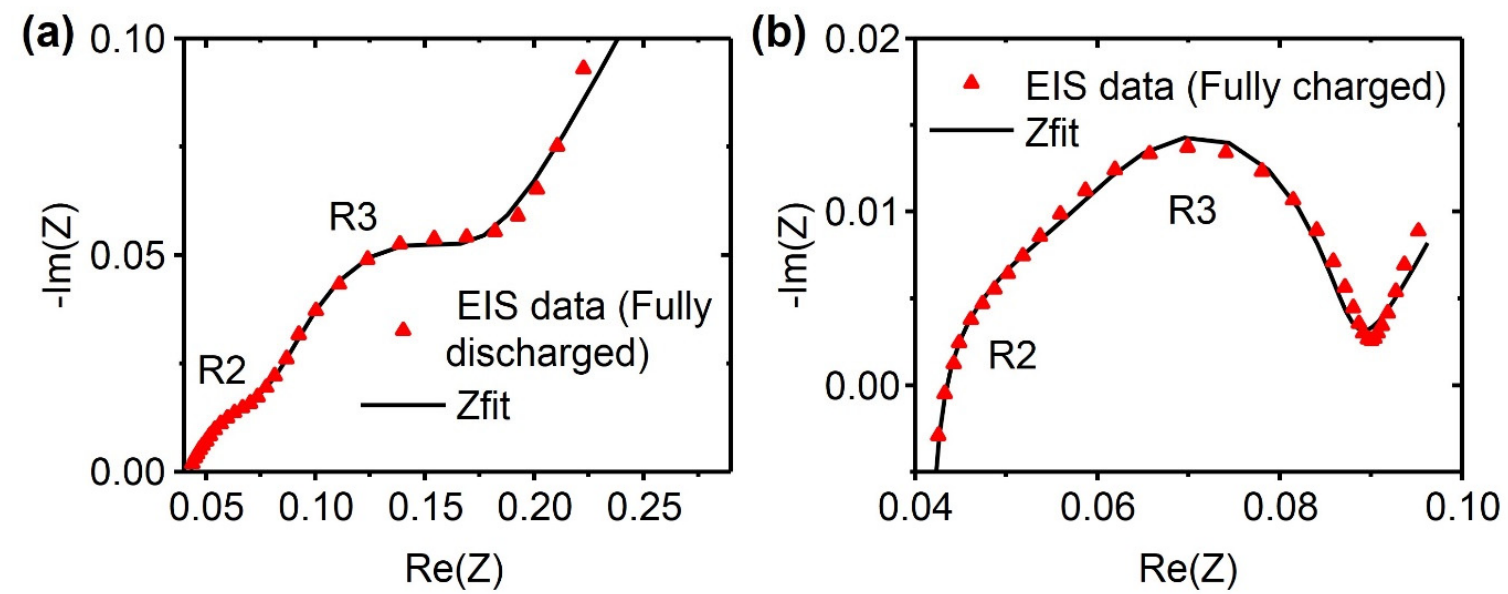

(c)

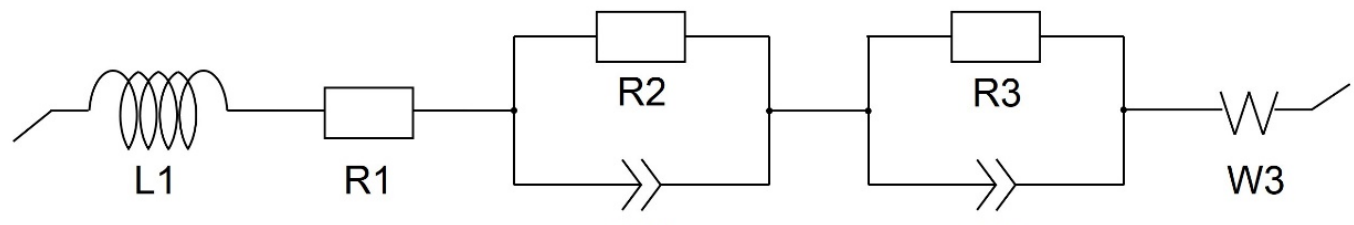

Q2

Q3

(d)

\begin{tabular}{cccc}
\hline Resistance $(\mathbf{m} \Omega)$ & R1 & R2 & R3 \\
\hline Fully Discharged & 40 & 50 & 52 \\
\hline Fully Charged & 40 & 30 & 18 \\
\hline
\end{tabular}

Figure S2. Nyquist plots of the fully (a) discharged (b) charged state of the LIB cell at frequencies ranging from $200 \mathrm{kHz}$ to $10 \mathrm{mHz}$ measured at room temperature $\left(25^{\circ} \mathrm{C}\right)$. (c) Simplified equivalent circuit of the LIB cell and (d) its resistances. The resistance values were obtained from $Z$-fit analysis. 


\section{Characterization of CNT-sensor according to the CNT thickness}
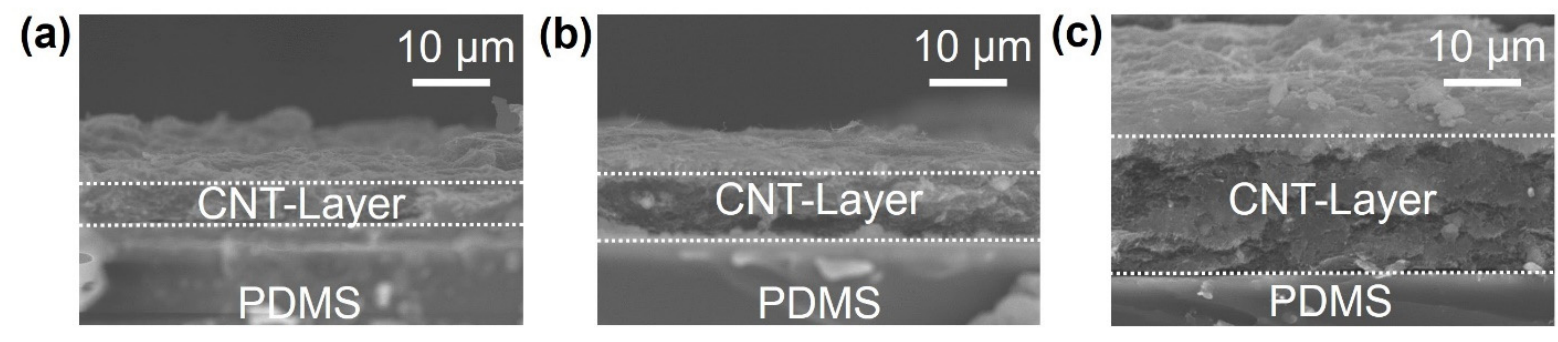

Figure S3. Cross-sectional SEM images of the sensor with different thickness of the CNTs. The thickness of the CNT-layer is controlled by changing the duration of spray-coating. It is noted that other conditions such as the concentration of CNT dispersion, the spray-coating pressure, and the size of shadow mask are identical for all fabricated sensors. The thickness of CNT-layer is measured to be 5.6, 9.1, and $18.1 \mu \mathrm{m}$ for the spray-coating time of (a) $0.5 \mathrm{~min}$, (b) $1 \mathrm{~min}$, and (c) $1.5 \mathrm{~min}$, respectively. 
(a)

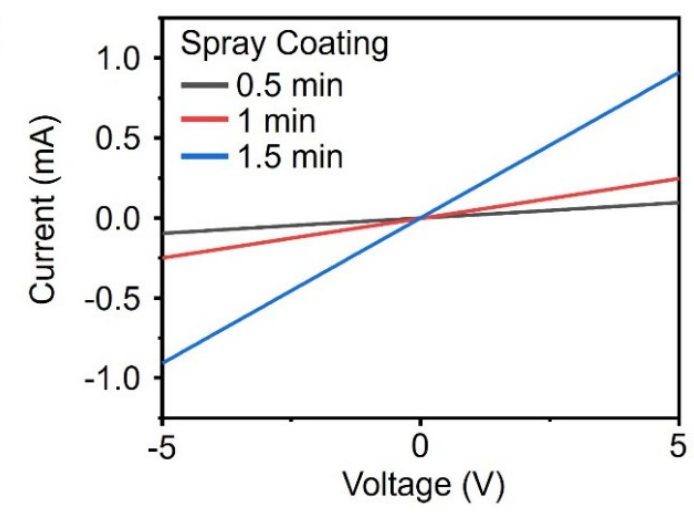

(b)

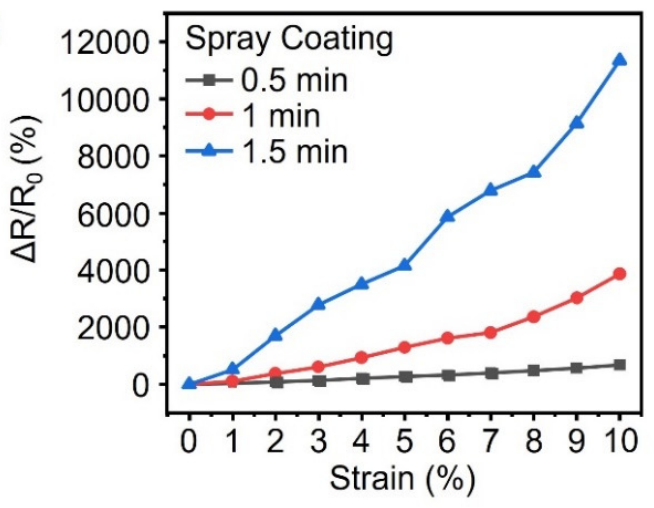

(c) Spray Coating: $0.5 \mathrm{~min}(\mathrm{t}=5.6 \mu \mathrm{m})$

$1 \mathrm{~min}(\mathrm{t}=9.1 \mu \mathrm{m})$

$1.5 \min (\mathrm{t}=18.1 \mu \mathrm{m})$

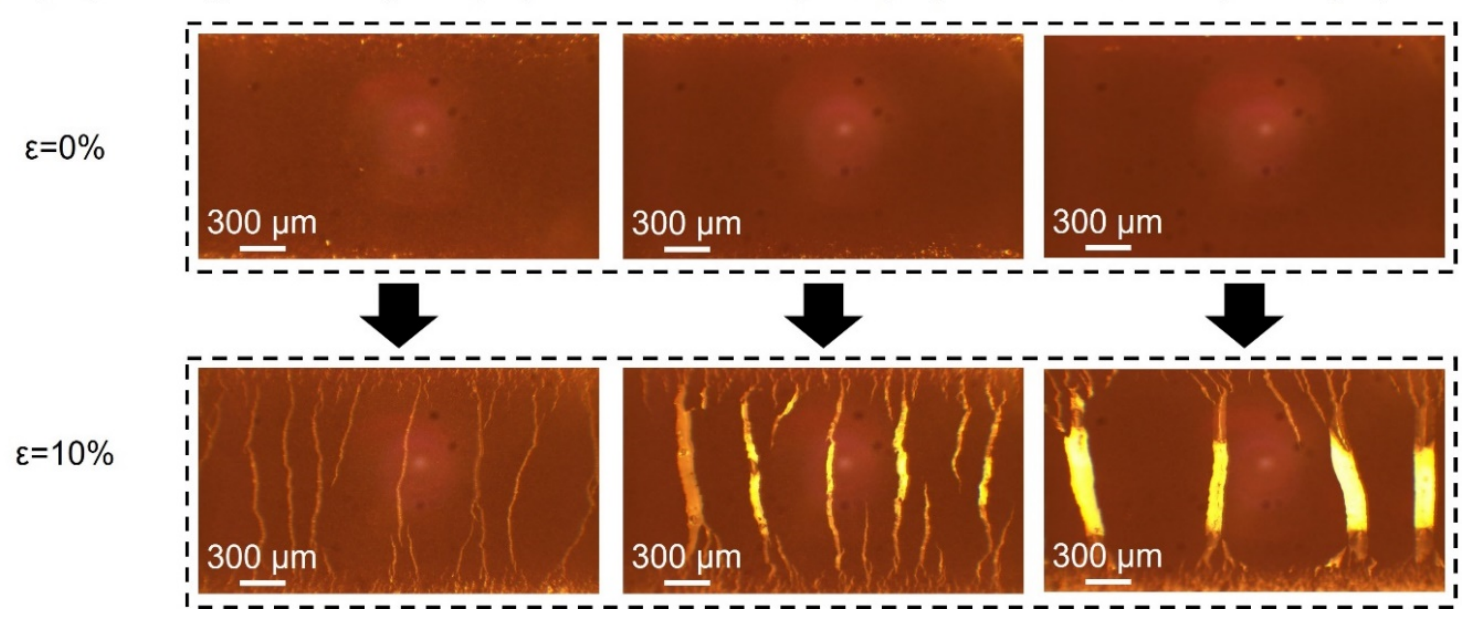

Figure S4. Characterizations of the CNT sensors with different CNT thickness. (a) I-V curves of the CNT-sensor with the different CNT thickness of 5.6, 9.1, and $18.1 \mu \mathrm{m}$. A thicker CNT-layer leads to lower electrical resistance. (b) Strain response of the CNT sensors with different CNT thickness of 5.6, 9.1, and $18.1 \mu \mathrm{m}$. A thicker CNTs exhibit a higher gauge factor in the identical range of applied tensile strain. It could be due to the lower initial resistance and more efficient load transfer by the densely entangled CNTs in the network. ${ }^{2-5}$ (c) Optical microscope images of the CNTs before and after applying 10\% tensile strain. Larger cracks are generated in the thicker CNTs under identical strain. 


\section{Mechanical and thermal reliability of CNT-sensor}

(a)

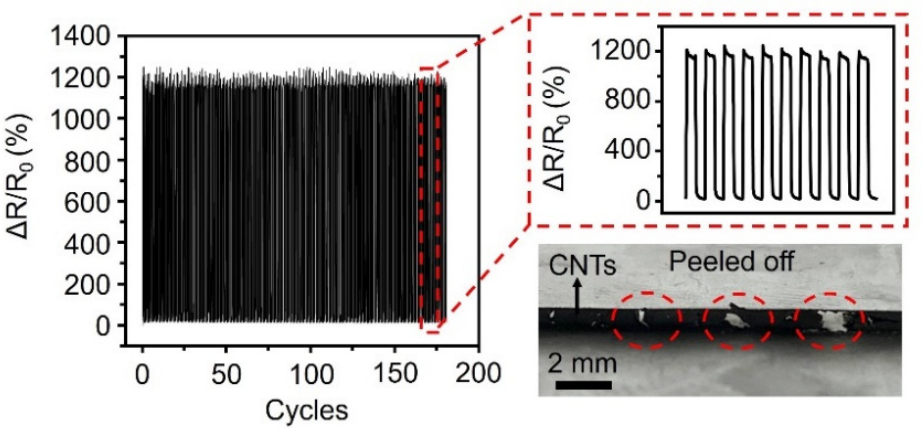

(b)

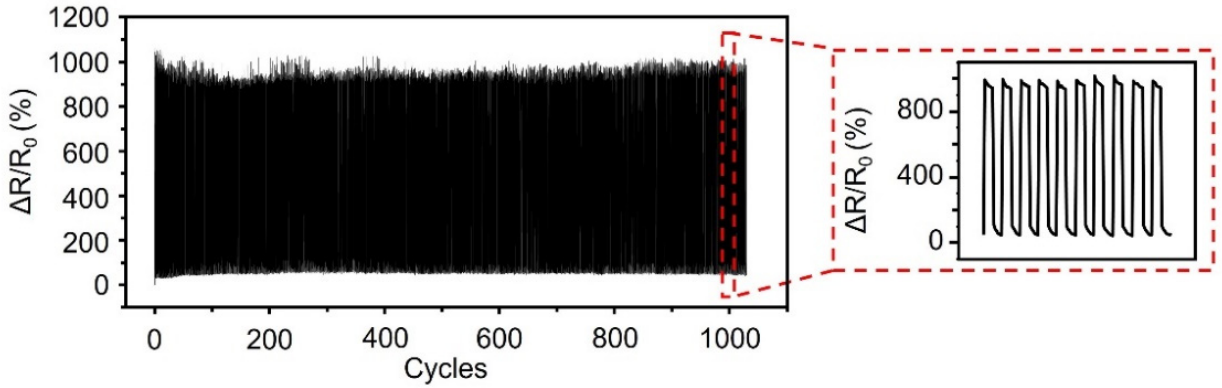

(c)

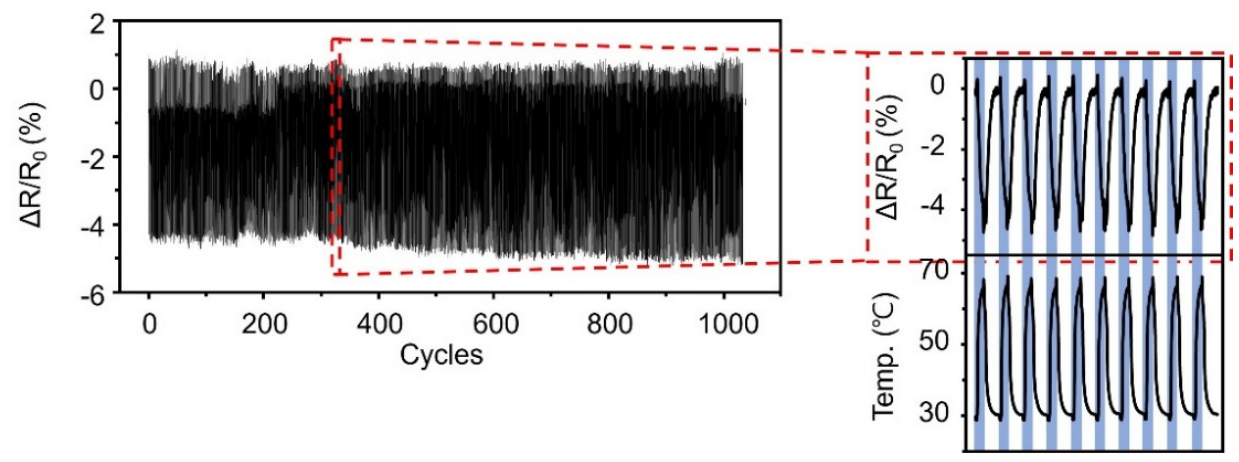

Figure S5. Long-term cyclic durability of the CNT-sensor measured under the repeated tensile strain. (a) Limited durability of the $18.1 \mu \mathrm{m}$-thick CNT-network that is prepared by $1.5 \mathrm{~min}$ of spray-coating. Although the thicker CNTs show a higher strain response, the durability is limited to 180 cycles under the strain of $2 \%$ due to excessively generated cracks in the CNT-network. Inset shows the optical microscope image of CNTs that are peeled off from the PDMS substrate. (b) High stability of $9.1 \mu \mathrm{m}$-thick CNT-network that is prepared by $1 \mathrm{~min}$ of spray-coating. The insignificant change in the resistance over 1030 cycles without noticeable degradation demonstrates the high reliability of CNT-sensor. Thus, the spraying time for the CNT coating is fixed to $1 \mathrm{~min}$ throughout the work in order to secure both sensitivity and durability. (c) Temperature stability is verified by exposing the CNT-sensor to the repeated temperature change from 29.2 to $69.2^{\circ} \mathrm{C}$ up to 1030 cycles. The custom-built thermal shock testing system was devised to expose the CNT-sensor to temperature change. The CNT-sensor stably responds to the temperature change, and its resistance is decreased as the temperature increases due to the negative TCR of the CNT-sensor. Slight Drift in signal could be due to the temperature instability of our custom-built experimental system. 
5. Dependence of the coefficient of thermal expansion of PDMS on temperature

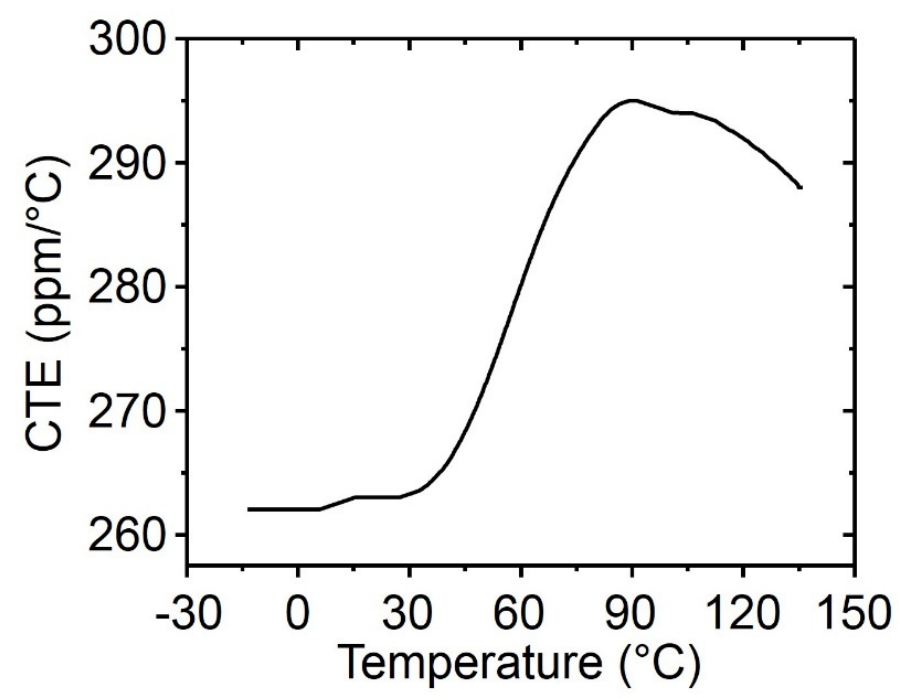

Figure S6. Dependence of coefficient of thermal expansion (CTE) on the temperature of PDMS (Sylgard 184 silicone elastomer) offered by Sandia National Laboratories. ${ }^{6}$ The CTE of PDMS starts increasing at $\sim 40{ }^{\circ} \mathrm{C}$ and reaches a maximum value of $296 \mathrm{ppm} /{ }^{\circ} \mathrm{C}$ at $90{ }^{\circ} \mathrm{C}$. Since the CNTsensor is sensitive to microscale tensile strain, the resistance of the CNT network increases owing to this thermal expansion. As such, a glass substrate with low thermal expansion coefficient was used to extract the TCR of the CNT network. 


\section{Noise processing using RLS algorithm}

Considering that all output and input data are determined at the sampling instant, the linear relationship between the voltage and current of the CNT-sensor can be formulated by the RLS algorithm as follows:

$$
y(t)=\varphi^{T}(t) \theta(t)
$$

where the estimation parameter $\theta(t)$, input regression $\varphi^{T}(t)$, and the measured output are given as $\theta(t)=R(t), \varphi^{T}(t)=I(t)$, and $y(t)=V(t)$, respectively. $R(t)$ is the equivalent resistance of the CNT-sensor, $I(t)$ is the constant current applied to the CNT-sensor, and $V(t)$ is the voltage measured at both ends.

The recursive process of the RLS algorithm can be described as: ${ }^{7}$

$$
\begin{aligned}
& \hat{\theta}(\mathrm{t})=\hat{\theta}(t-1)+K(t)\left(y(t)-\varphi^{T}(t) \hat{\theta}(t-1)\right) \\
& K(t)=P(t-1) \varphi(t)\left[\lambda I+\varphi^{T}(t) P(t-1) \varphi(t)\right]^{-1} \\
& P(t)=\lambda^{-1}\left[I-K(t) \varphi^{T}(t)\right] P(t-1)
\end{aligned}
$$

where $I$ is the identity matrix, and $K(t)$ and $P(t)$ are the Kalman gain and covariance matrices, respectively.

To cope with the time-varying properties in the measurement system of the CNT-sensor, the weighted least squares criterion is handled by putting less weight on the older measurements. Therefore, the weighting function is set to:

$$
\Gamma(t, k)=\lambda^{t-k} ; \text { i.e., } \lambda(t)=\lambda
$$

where the choice of the forgetting profile $\Gamma(t, k)$ is conceptually simple. It is common to select this profile so that the least square criterion weighting essentially contains those measurements that are relevant to the current properties of the system. For a system that changes gradually and in a "stationary manner", the most common choice is to take a constant forgetting factor, such as Equation (3). The forgetting factor $\lambda$ is always chosen to be a positive constant slightly smaller than one, so that $\Gamma(t, k)=e^{(t-k) \log \lambda} \approx e^{(t-k)(1-\lambda)}$. This means that measurements that are older than $T_{0}=1 /(1-\lambda)$ samples are included in the criterion with a weight that is $e^{-1} \approx 36 \%$ of that of the most recent measurements. It is called a "memory time constant" of the criterion and represented as:

$$
T_{0}=(1-\lambda)^{-1}
$$

If the system remains approximately constant over $T_{0}$ samples, a suitable choice of $\lambda$ can be made from Equation (4). Typical choices of $\lambda$ are in the range between 0.95 and 0.999 . The smaller the value of $\lambda$, the less weight is assigned to the older data; i.e., the past data are forgotten faster. In this work, $\lambda \sim 0.95$ was selected to make a reasonable trade-off between tracking ability and noise sensitivity. 


\section{LIB swelling during regular charge and discharge cycles}

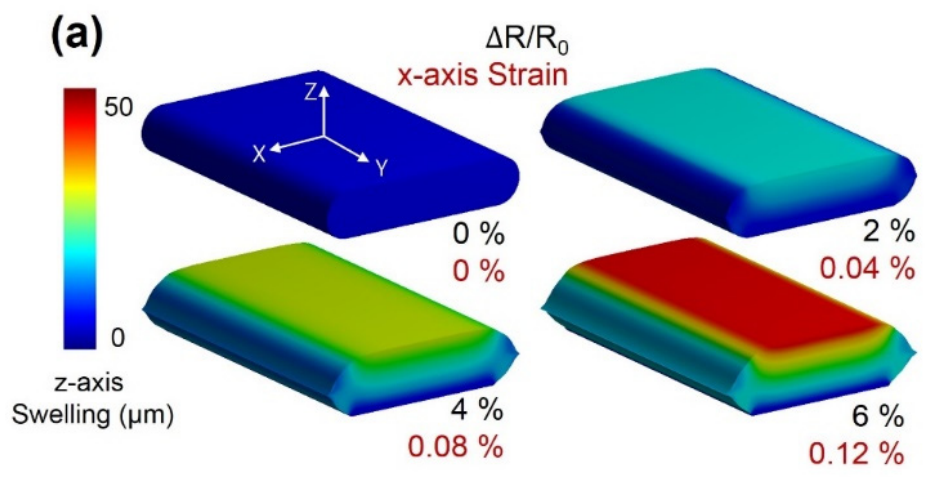

(b)

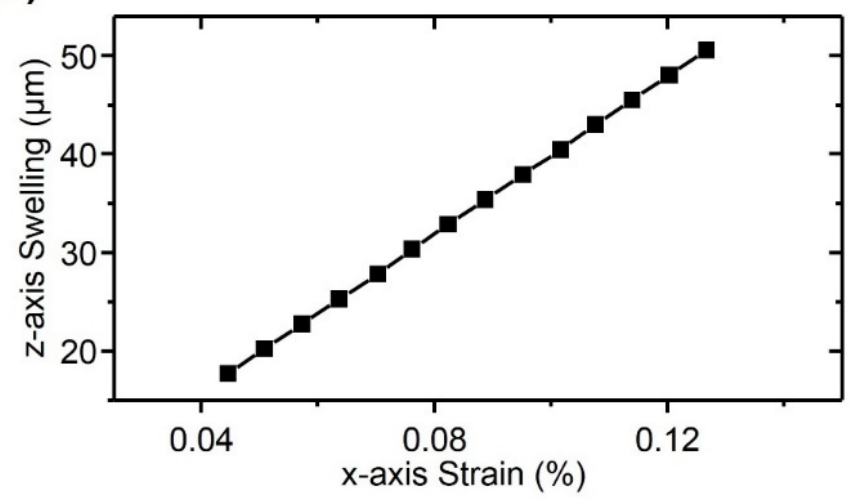

Figure S7. (a) FEM was used to determine the amount of LIB swelling ( $z$-axis displacement) generated during the charge and discharge cycles. The CNT-sensor was mounted parallel to the $x$ axis, and its resistance increased up to $\sim 6 \%$ while charging. Considering that the measured gauge factor was $50(\Delta \varepsilon<1 \%)$, the tensile strain in the longitudinal direction (i.e., $x$-axis direction) was calculated to be $\sim 0.12 \%$. The pressure inside the LIB was adjusted to match the corresponding $x$ axis strain, and the $z$-axis swelling at this moment was obtained, which had increased to $50 \mu \mathrm{m}$. (b) Linear relationship between the $x$-axis strain and the $z$-axis swelling analyzed by FEM. 


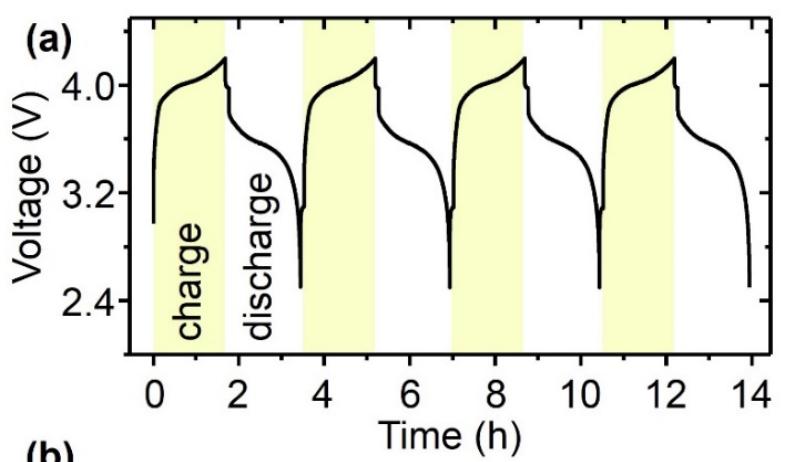

(b)
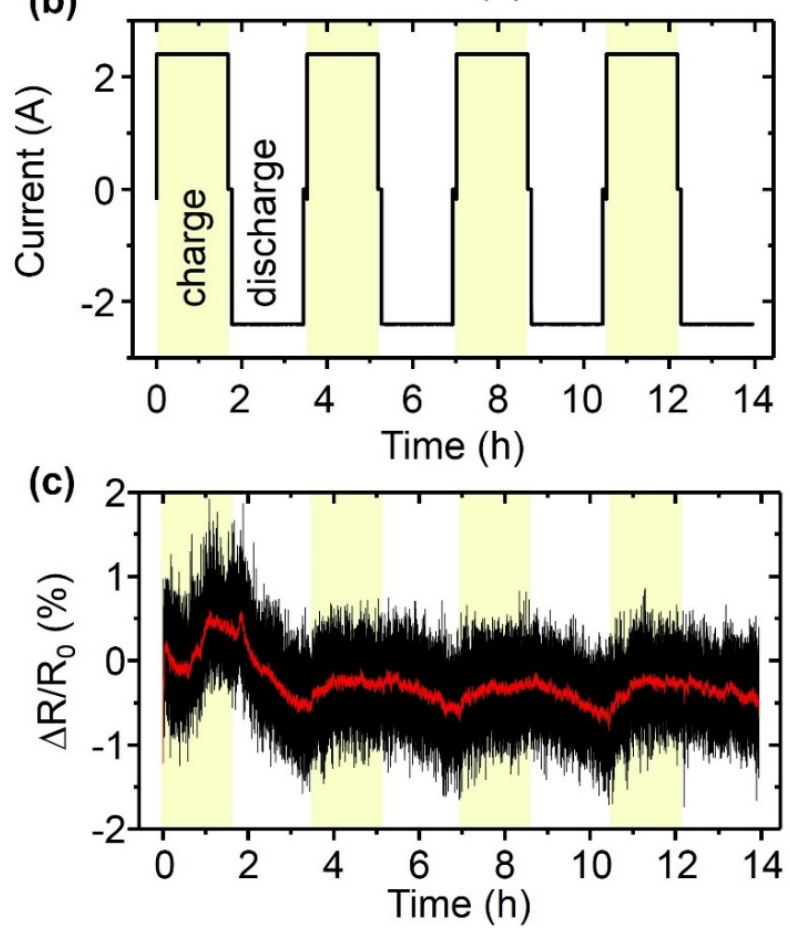

Figure S8. To confirm the reproducibility of our approach, a different set of LIB and CNT-sensor was prepared and the swelling was successfully measured during $0.4 \mathrm{C}$-rate charge and discharge cycles. (a) Voltage and (b) current of LIB for charge-discharge cycles. (c) Changes in the relative resistance of the CNT-sensor corresponding to the charge and discharge cycles of the LIB. The increased resistance during charging indicates the increased swelling, similar to the result shown in Figure 4(b) in the main text. The RLS algorithm was also applied for signal processing (red solid line). It is worth noting that the amount of swelling can be different depending on the performance and status of the LIB, but the applicability of CNT-sensor can be verified. 


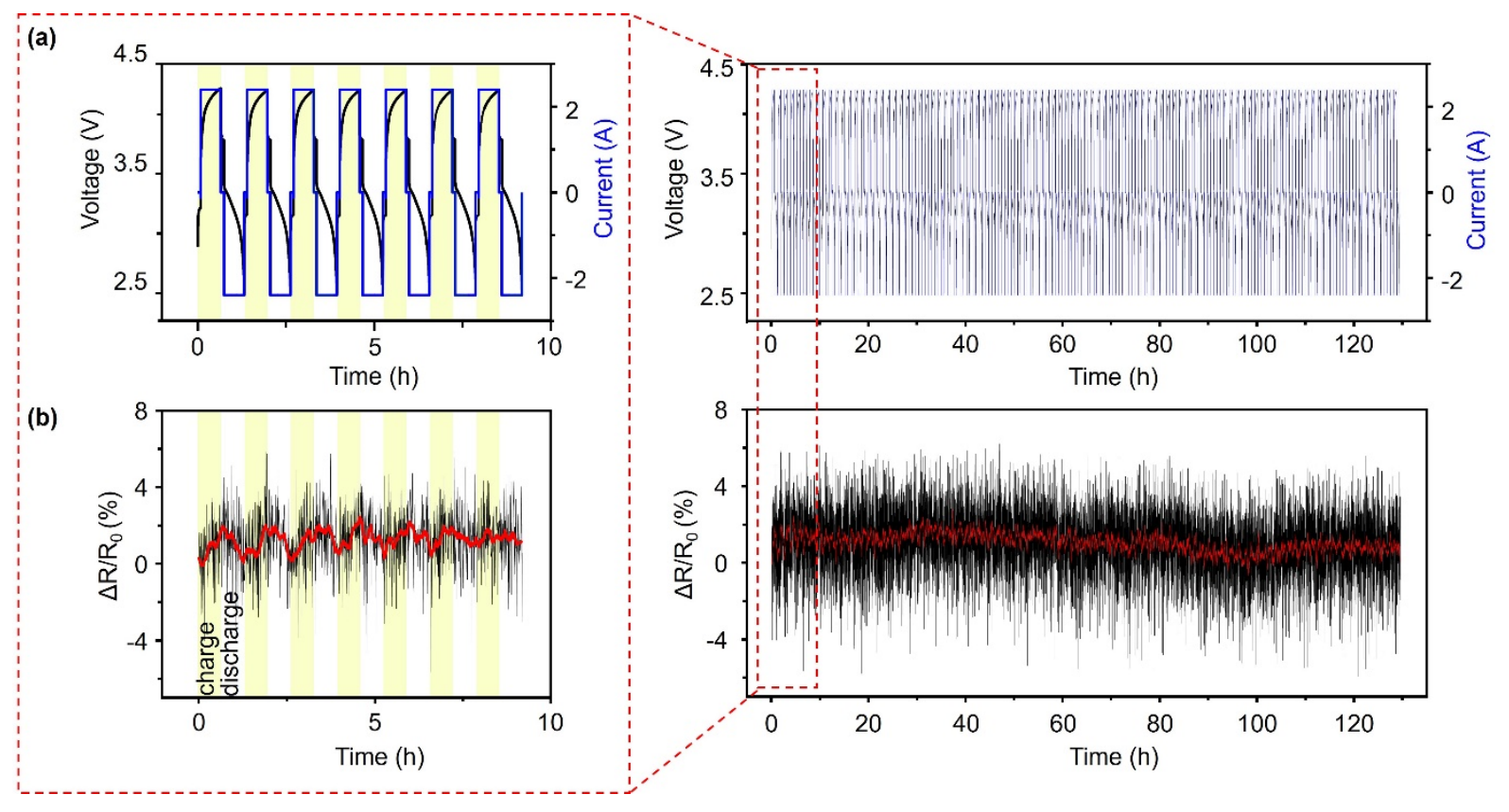

Figure S9. In-situ measurement of LIB swelling during regular charge and discharge over 100 cycles. (a) Charge-discharge cycles of LIB and (b) Corresponding resistance change of the CNTsensor. 
8. Irregular, irreversible, thermomechanical swelling of LIB

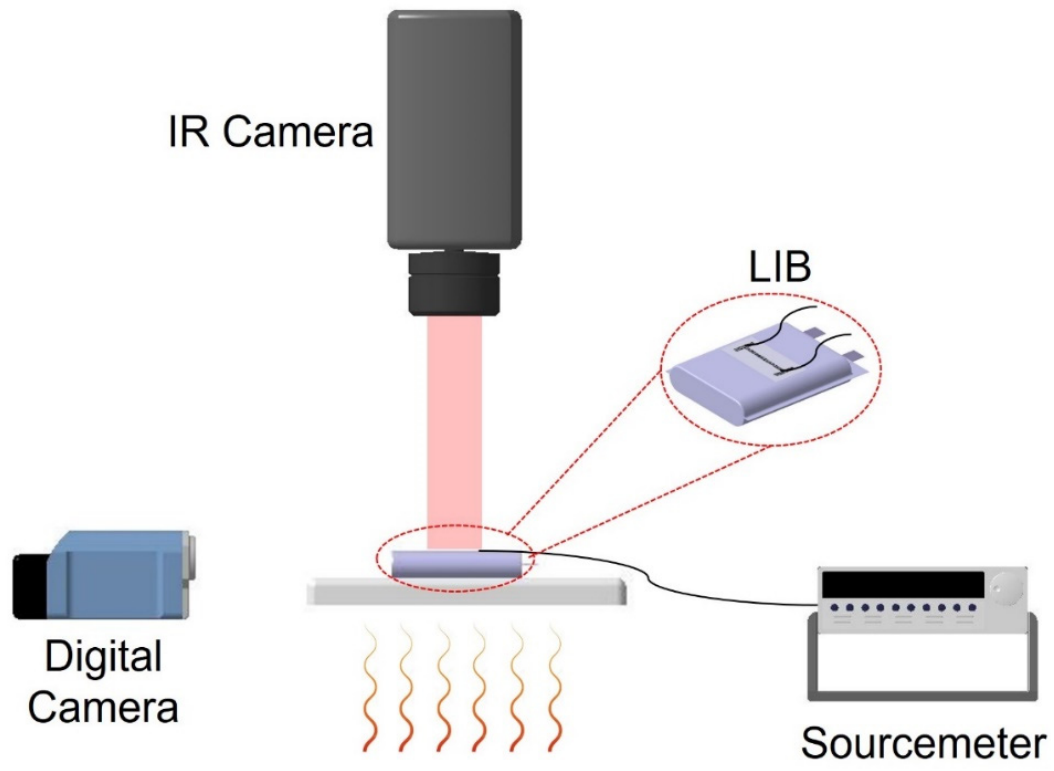

Figure S10. Schematic of the experimental setup for measuring the irreversible thermomechanical swelling. ${ }^{8}$ The LIB with an integrated CNT-sensor is exposed to an elevated temperature. Digital camera and IR camera capture the swelling of the LIB and the change in temperature of the CNTsensor, respectively. Simultaneously, the change in the resistance of the CNT-sensor is measured and calibrated with the measured temperature change. 

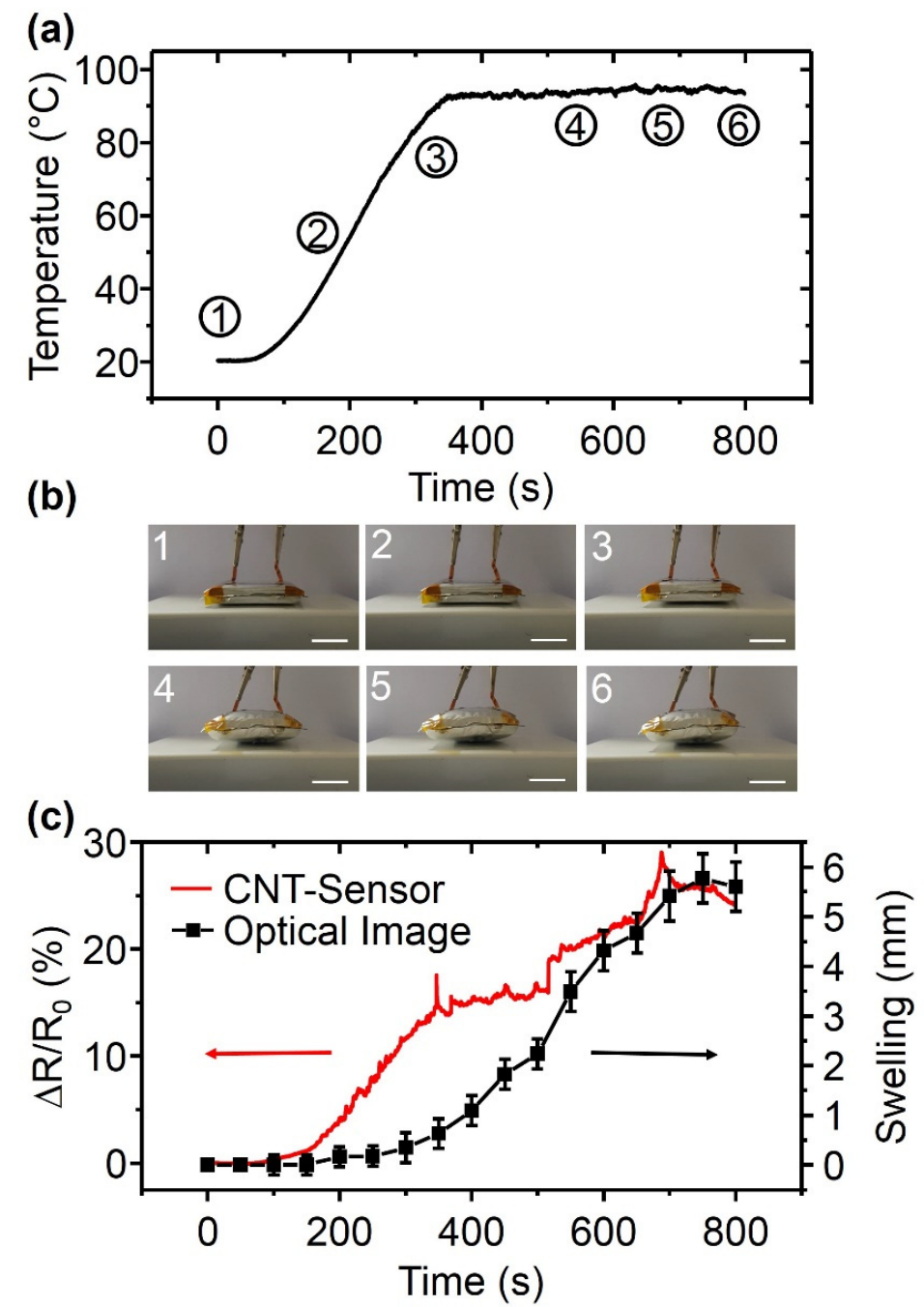

Figure S11. Reproducibility of the irregular, irreversible swelling measurements was verified with different sets of LIB and CNT-sensor. (a) The CNT-sensor-mounted LIB was placed on a hot plate, and the temperature of hot plate was set to $220^{\circ} \mathrm{C}$. The temperature of the CNT-sensor increased up to $95^{\circ} \mathrm{C}$. (b) Photographs of the swollen LIB with the integrated CNT-sensor. Number in each panel corresponds to the number shown in (a). All scale bars are $2 \mathrm{~cm}$. (c) Change in relative resistance of the CNT-sensor and the measured swelling of LIB from the optical images. From 200 to $400 \mathrm{~s}$, the CNT-sensor sensitively captured the LIB swelling, which was difficult to distinguish by the optical images. The volume shrinkage of the LIB at $\sim 700 \mathrm{~s}$ due to gas leakage was also measured by the CNT-sensor. 


\section{References}

(1) Johnston, I. D.; McCluskey, D. K.; Tan, C. K. L.; Tracey, M. C. Mechanical Characterization of Bulk Sylgard 184 for Microfluidics and Microengineering. J. Micromech. Microeng. 2014, 24, 035017.

(2) Song, X.; Liu, S.; Gan, Z.; Lv, Q.; Cao, H.; Yan, H. Controllable Fabrication of Carbon Nanotube-Polymer Hybrid Thin Film for Strain Sensing. Microelectron. Eng. 2009, 86, 23302333.

(3) Lee, D.; Hong, H. P.; Lee, C. J.; Park, C. W.; Min, N. K. Microfabrication and Characterization of Spray-Coated Single-Wall Carbon Nanotube Film Strain Gauges. Nanotechnology 2011, 22, 455301

(4) Wang, X.; Sparkman, J.; Gou, J. Strain Sensing of Printed Carbon Nanotube Sensors on Polyurethane Substrate with Spray Deposition Modeling. Compos. Commun. 2017, 3, 1-6.

(5) Wang, X.; Li, J.; Song, H.; Huang, H.; Gou, J. Highly Stretchable and Wearable Strain Sensor Based on Printable Carbon Nanotube Layers/Polydimethylsiloxane Composites with Adjustable Sensitivity. ACS Appl. Mater. Interfaces 2018, 10, 7371-7380.

(6) Classes of Polymers, Sandia National Laboratories, www.sandia.gov/polymer-properties (accessed December 10, 2019).

(7) L. Lennart, System Identification: Theory for the User, 2nd ed.; Prentice Hall: Upper Saddle River, NJ, USA, 1999.

(8) Choi, W.; Seo, Y.; Yoo, K.; Ko, T. J.; Choi, J. Carbon Nanotube-Based Strain Sensor for Excessive Swelling Detection of Lithium-Ion Battery. 20th Int. Conf. Solid-State Sensors, Actuators Microsystems (Transducers 2019), Berlin, Germany, June 2019, 2356-2359. 